\title{
On the Feasibility of Utilizing Smartphones for Vehicular Ad Hoc Networking
}

\author{
Wim Vandenberghe, Ingrid Moerman, Piet Demeester \\ Internet Based Communication Networks and Services \\ Ghent University \\ Ghent, Belgium \\ Wim.vandenberghe@intec.ugent.be
}

\begin{abstract}
Last years, a lot of research was performed focusing on cooperative road safety applications such as nearby traffic jam warning, approaching emergency vehicle warning, road obstacle warning, wrong way driver warning, etc. The high cost of the required in-vehicle systems however seems to obstruct end-user adoption. An alternative could be to utilize smartphones for vehicular ad hoc networking. From a business point of view this seems attractive because smartphones already enjoy a successful user adoption, and they provide most of the hardware required for cooperative road safety applications. However, from a technical point of view there is one aspect of the smartphone approach that could prove to be an insurmountable problem: these phones are equipped with standard IEEE $802.11 \mathrm{a} / \mathrm{b} / \mathrm{g}$ (WiFi) wireless interfaces, instead of the automotive variant IEEE 802.11p. In this paper, a measurement campaign is presented that investigates the technical feasibility of the smartphone approach.
\end{abstract}

\section{Keywords: VANET, IEEE 802.11p, Smartphone}

\section{INTRODUCTION}

Recently, a significant amount of research was performed that focuses on cooperative road safety applications such as nearby traffic jam warning, approaching emergency vehicle warning, road obstacle warning, wrong way driver warning, intersection collision warning, etc [1]. In literature it is estimated that such applications can result in a decrease of accidents with injuries or fatalities of $20 \%$ to $50 \%$ [2]. The common understanding in research is that the communication between vehicles will be performed using a local wireless communication technology based on IEEE 802.11, the 802.11p amendment. This technological choice is mainly driven by the assumption that local communication is more likely to meet the stringent delay requirements of the safety applications. The common research view is that in the future, dedicated OEM onboard units with IEEE 802.11 p communication capabilities will be installed in new vehicles.

One of the pitfalls of this technical approach is the well known chicken and egg problem: users will only buy the systems if an immediate positive safety impact is offered, but to achieve that positive impact first many vehicles have to be equipped with the systems. This user adoption problem is amplified by the end-user cost of such systems. The on board unit (OBU) for cooperative road safety applications will be very similar to current built-in car navigation systems. The reason is that both systems roughly consist of the same components (central processing unit, GPS based localization services, visual and auditive communication means towards the driver and road maps data). Price lists of several popular car models have been investigated. It can be concluded that in general prices of built-in car navigation systems vary between 1000 and 3000 euro. It can be assumed that future OBUs for cooperative road safety applications will cost about the same.

This rather high cost for the end-user is in sharp contrast with the fact that the willingness-to-pay for cooperative road safety applications is low, even for applications that are considered useful by the motorists [3], [4]. This is problematic to achieve a successful user adoption. Another challenge lies in the fact that the decision to equip the vehicle with cooperative safety systems has to be made at the time of vehicle purchase. Because of the long life span of vehicles, this can further hinder the successful uptake of these systems by end-users.

To overcome these problems, the utilization of smartphones for vehicular ad hoc networking could be a possible solution. Both the Apple iOS and the Android OS are very popular smartphone operating systems, with a daily growing user base. In November $2010,31 \%$ of all US consumers already owned a smartphone, and $45 \%$ of recent acquirers chose a smartphone over a feature phone [5]. From a hardware point of view, smartphones provide most of the required hardware components: a powerful central processing unit, a GPS receiver, an accelerometer, a color touchscreen and a pair of speakers. To use these devices for cooperative road safety, only appropriate mobile applications have to be purchased by the end-user, resulting in a negligible cost. However, from a technical point of view there is one aspect of the smartphone approach that could prove to be an insurmountable problem: these phones are equipped with standard IEEE $802.11 \mathrm{a} / \mathrm{b} / \mathrm{g}$ $(.11 \mathrm{a} / \mathrm{b} / \mathrm{g})$ wireless interfaces, instead of the automotive variant IEEE $802.11 \mathrm{p} \mathrm{(.11p).} \mathrm{It} \mathrm{is} \mathrm{unknown} \mathrm{if} \mathrm{these} \mathrm{standard} .11 \mathrm{a} / \mathrm{b} / \mathrm{g}$ interfaces can meet the performance criteria to be suitable for vehicular ad hoc networking. This open question is the focal point of this paper. In section II the technical challenges for smartphone vehicular ad hoc networks (VANETs) are introduced, while in sections III and IV the results of different measurement campaigns are presented. Conclusions are drawn in section $\mathrm{V}$. 


\section{CHALLENGES WHEN UTILIZING SMARTPHONES FOR VEHICULAR AD HOC NETWORKING}

As explained in the introduction, smartphones are equipped with standard IEEE $802.11 \mathrm{a} / \mathrm{b} / \mathrm{g}$ wireless interfaces instead of the automotive variant IEEE $802.11 \mathrm{p}$. This .11p standard is based on the OFDM physical layer of the .11a standard, enhanced with several optimizations to improve performance in VANETs [6], [7]. To achieve a robust connection under high velocities, the PHY layer uses half clocked mode with $10 \mathrm{MHz}$ channels instead of the standard $20 \mathrm{MHz}$. As a result, parameters in the time domain are doubled and data rates are halved. These measures make the signal more robust: effects of Doppler spread are reduced because of the reduced bandwidth, and the larger guard interval reduces inter-symbol interference caused by multi-path propagation. To provide larger communication ranges for vehicular scenarios, the maximum allowable Effective Isotropic Radiated Power (EIRP) is also raised to $33 \mathrm{dBm}(2 \mathrm{~W})$ for most safety relevant messages. This results in a realistic communication range of 750 meters [8]. Finally, to avoid interference from non-VANET equipment, dedicated operating frequency bands are used (5.9 GHz band).

All these optimizations are not available to smartphones. This can negatively influence the VANET communication link performance in several ways. First of all, because of the lack of a dedicated channel, communication can be interfered by other devices operating in the same license free bands. The useful communication range will also be smaller because of the deteriorated signal quality, caused by different factors. One of them is the fact that the maximum allowable EIRP is an order of magnitude smaller in the smartphone approach. The specifications of several $.11 \mathrm{a} / \mathrm{b} / \mathrm{g}$ chipsets for mobile devices were inspected (Broadcom BCM4329, Texas Instruments WiLink, Atheros ROCm and GainSpan GS1011M), and in all cases EIRP values ranged from 15 to $19 \mathrm{dBm}$ (approx. 30 to 80 $\mathrm{mW}$ ). The fact that the internal antennas of the smartphone will be placed in the vehicle instead of on the roof of the vehicle also negatively influences the signal quality. Besides, the usage of $20 \mathrm{MHz}$ channels makes the signal more sensitive to Doppler shift and multi-path fading.

Based on the above, it can be concluded without a doubt that communication link performance will be worse when utilizing smartphones for VANETs instead of IEEE 802.11p hardware. However, it is uncertain if the deteriorated performance will still be sufficient to support vehicular ad hoc networking in an acceptable manner. At the time of writing and to the best of our knowledge, no studies could be found in literature that provide insights in this specific problem.

\section{INFLUENCE OF NON-DEDICATED CHANNEL}

As mentioned in section II, ad hoc networks based on smartphones can be interfered by other devices operating in the same license free bands. To gain insights in the magnitude of this problem, measurements with a spectrum analyzer (Fluke networks AirMagnet Spectrum XT) were performed on public roads. The external antenna used for the measurements was attached to the middle of the car windscreen using a suction cup holder. This mimics the placement of smartphones in VANET scenarios. Two different kinds of graphs were recorded: spectrum density graphs and channel duty cycle vs. time graphs. The former shows the popularity of a particular channel over time: the $\mathrm{X}$-axis shows the channel, the $\mathrm{Y}$-axis shows the minimum and maximum power readings in $\mathrm{dBm}$. This is helpful to identify infrequent transmitters. The latter displays how often an interfering signal is present over time. A high duty cycle means an interferer is constantly transmitting and will most certainly cause problems on the affected channel.

Three different environments were investigated (highway, rural and urban), and for each of them both the characteristics in the $2.4 \mathrm{GHz}$ and the $5.5-5.7 \mathrm{GHz}$ band were recorded. The results are presented in the following subsections.

\section{A. Highway scenario}

The highway environment was characterized by measuring a trajectory of approximately $50 \mathrm{~km}$ on the E40 highway between Ghent and Brussels (Belgium). The results are depicted in Figure 1 - Figure 4. It can be seen that in both the 2.4 and the $5.5 \mathrm{GHz}$ band the level of interference is very low. In Figure 2 some peaks in the duty cycle of the $2.4 \mathrm{GHz}$ band can be seen in the beginning of the trajectory, which corresponds with the more urban environment when driving on the highway approach. As soon as the highway entered more isolated terrain, no interference activity was further noticed. The only exception is one very short peak over the entire 50 $\mathrm{km}$ trajectory. On the $5.5 \mathrm{GHz}$ band no interference could be identified at all. It can be concluded that in general interference from other mobile devices can be neglected, and that from an interference point of view, both IEEE $802.11 \mathrm{~g}$ communication on the $2.4 \mathrm{GHz}$ band and IEEE 802.11a communication on the $5.5 \mathrm{GHz}$ band can be considered as feasible for VANETs in the highway scenario.

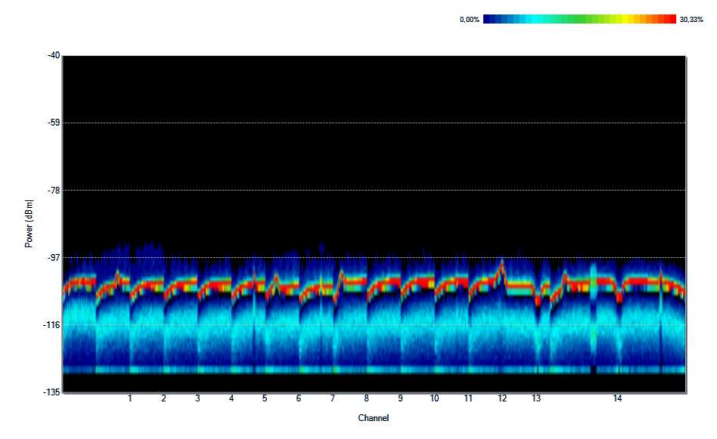

Figure 1: Highway spectrum density $2.4 \mathrm{GHz}$

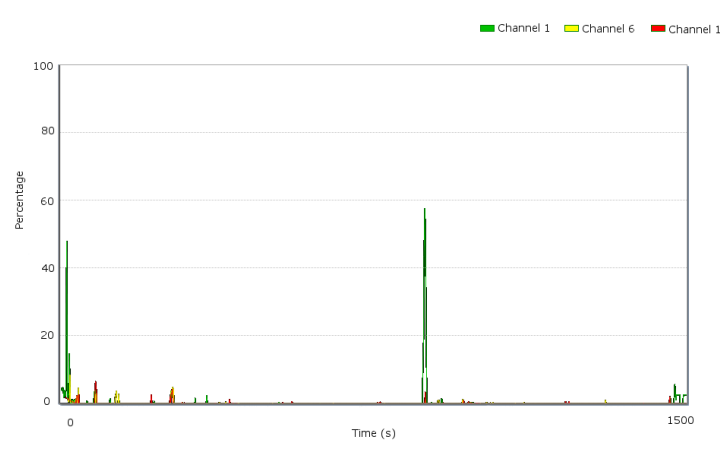

Figure 2: Highway duty cycle over time $2.4 \mathrm{GHz}$ 


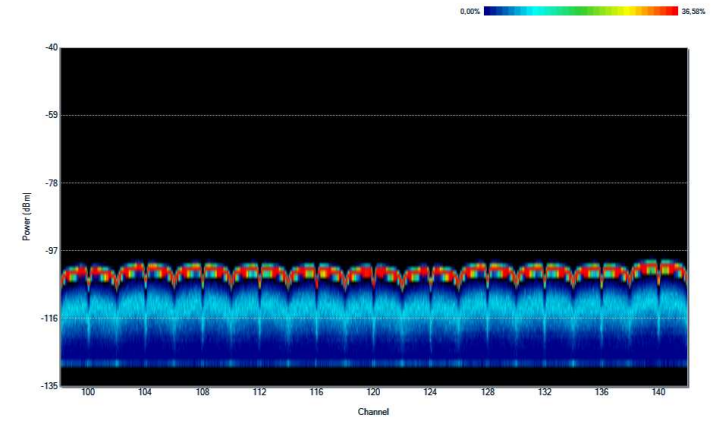

Figure 3: Highway spectrum density $5.7 \mathrm{GHz}$

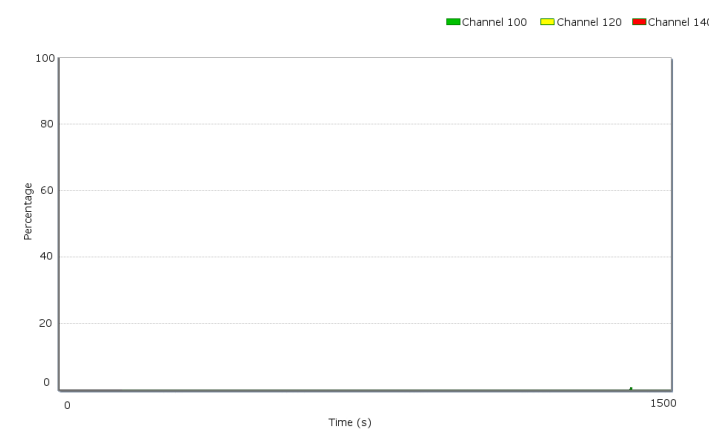

Figure 4: Highway duty cycle over time $5.7 \mathrm{GHz}$

\section{B. Rural scenario}

The rural environment was characterized by measuring a trajectory of approximately $25 \mathrm{~km}$ on the N9 road between Ghent and Alost. However, the results were equal to the urban measurements. This is because ribbon development was found almost over the entire trajectory. This form of spatial planning is very common in Belgium. Because of the limited value of depicting the same results twice, we refer the reader interested in the rural scenario to the urban scenario presented section $\mathrm{C}$.

\section{Urban scenario}

The urban environment was characterized by measuring a trajectory of approximately $10 \mathrm{~km}$ in the city centre of Ghent. The results are depicted in Figure 5 - Figure 9. Similar to the highway environment, no significant interference could be identified on the $5.5 \mathrm{GHz}$ band. However, on the $2.4 \mathrm{GHz}$ band the interference was quite obvious, both on the spectrum density graph and the duty cycle graph. Figure 6 shows the duty cycle over time, on every channel continuous activity can be seen between 0 and $20 \%$, with regular peaks up to $100 \%$. This interference is not only caused by Wi-Fi devices, but also by other devices such as DECT phones and microwave ovens (Figure 7). It can be concluded that from an interference point of view, IEEE 802.11a is a suitable technology for urban VANET communication, but IEEE $802.11 \mathrm{~g}$ is not.

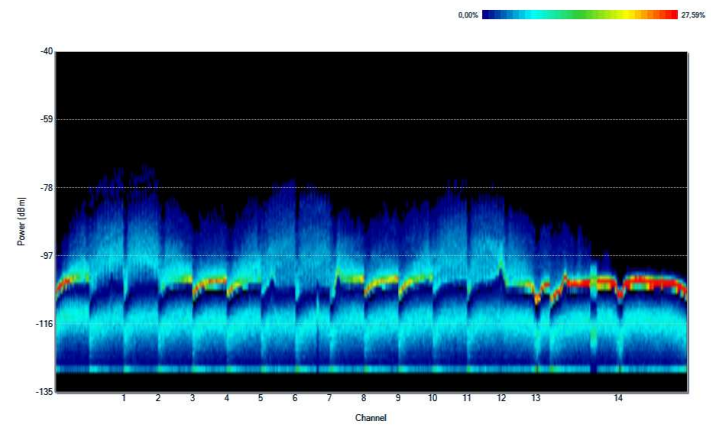

Figure 5: Urban spectrum density $2.4 \mathrm{GHz}$

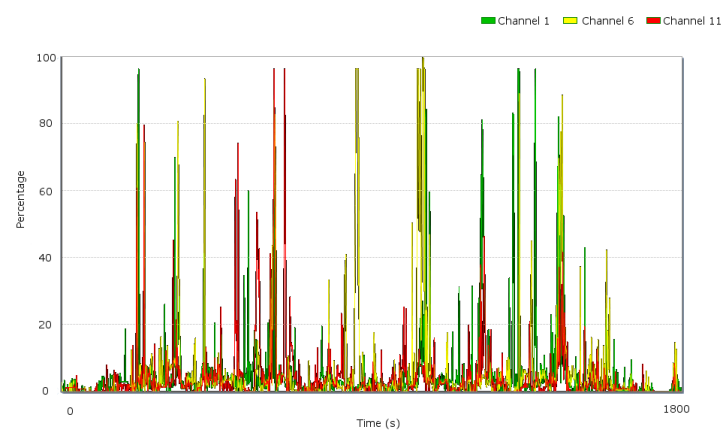

Figure 6: Urban duty cycle over time $2.4 \mathrm{GHz}$

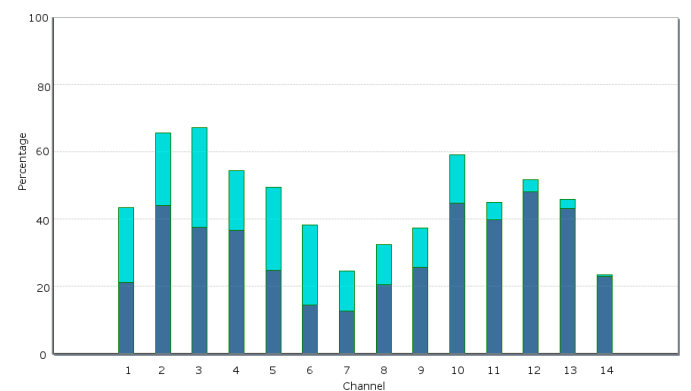

Figure 7: Urban duty cycle $2.4 \mathrm{GHz}$ - Classification of wireless activity at given moment in time

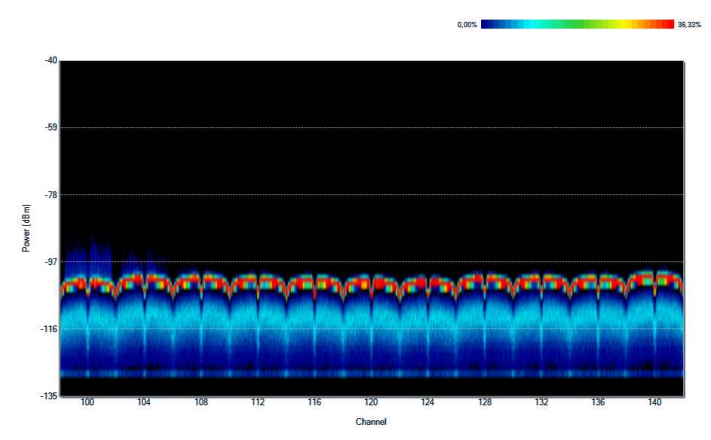

Figure 8: Urban spectrum density $5.7 \mathrm{GHz}$ 


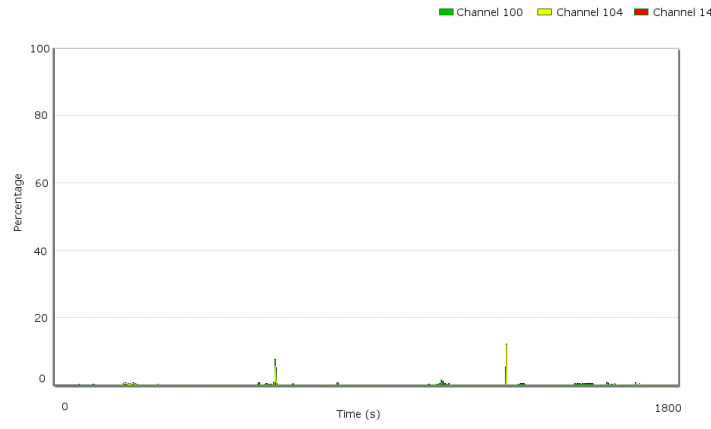

Figure 9: Urban duty cycle over time $5.7 \mathrm{GHz}$

\section{INFLUENCE OF DETERIORATED SIGNAL QUALITY}

As explained in section II it is expected that communication link performance will be worse when utilizing smartphones for VANETs instead of IEEE 802.11p hardware. This deterioration is caused by several characteristics of wireless communication. In the next subsections we will try to evaluate the individual effect of them. This knowledge about the wireless behavior in the envisaged scenario will then be brought together in the analysis of the final experiments.

\section{A. Description of experiments}

All executed link tests were performed with two vehicles on public roads. For one measurement, a test packet is broadcasted by the sender node. This ensures that there are no MAC retransmissions. The test packet contains an id, timestamp, sender location, and meaningless padding data to achieve a packet size of 300 bytes. This way the measured communication behavior will be applicable for cooperative awareness messages (CAM) [9] and decentralized environment notification messages (DENM) [10]. The receiving node completes the packet with its own location, and sends it back to the sender. The sending node processes the received response, and $\log _{s}$ the following parameters: id, signal to noise ratio, round trip time and distance between the sender and the receiver. As depicted in Figure 10, three different relative speeds were taken into account. In every scenario both $.11 \mathrm{~g}$ and .11a communication was tested using a standard Compex WLM54SAG 200mW IEEE $802.11 \mathrm{a} / \mathrm{b} / \mathrm{g}$ miniPCI card installed on a PC Engines Alix 3D3 motherboard. The antenna of the card was again attached inside the car to the middle of the windscreen using a suction holder. The data rate in both cases was $6 \mathrm{Mbps}$, corresponding with the more robust BPSK $1 / 2$ modulation. Transmit powers of 15,19 and $23 \mathrm{dBm}$ were applied, the first two values represent common smartphone specifications, while the last value indicates the possible performance gain of more powerful transmitters.

\section{B. Free Space Path Loss}

Free-space path loss (FPSL) is the loss in signal strength of an electromagnetic wave that would result from a line-of-sight path through free space, with no obstacles nearby to cause reflection or diffraction. It is proportional to the square of the distance between the transmitter and receiver, and also proportional to the square of the frequency of the radio signal. The latter is caused by the effect of the receiving antenna's aperture, which describes how well an antenna can pick up power from an incoming electromagnetic wave. This is dependent on wavelength [11]. Hence the higher the frequency, the higher the FPSL over the same distance, and thus the lower the communication range. This effect is illustrated in Figure 11, where the packet success rate (PSR) of the $.11 \mathrm{~g}$ and $.11 \mathrm{a}$ measurements are depicted for the front-front oriented approach at $40 \mathrm{~km} / \mathrm{h}$. This scenario avoids the effects of mobility and vehicle penetration as much as possible. We define useful communication range as the maximum range were a PSR of more than $66 \%$ is achieved, emphasized in the figure with a horizontal green line. On the left bank of the figure it can be seen that due to FPSL the useful range in the 2.4 GHz band is larger than in the $5.5 \mathrm{GHz}$ band. The difference however is not significant.

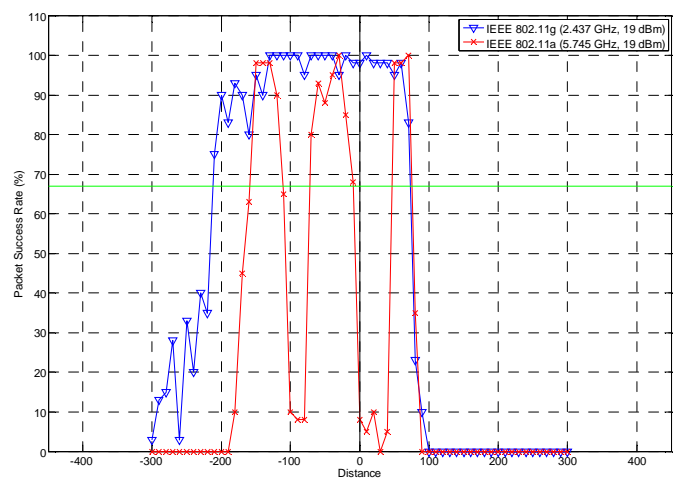

Figure 11: PSR frontal approach at $40 \mathrm{~km} / \mathrm{h}$

\section{Shadow fading}

Shadow fading occurs when an obstacle gets positioned between the wireless device and the signal transmitter [12]. This interference causes significant reduction in signal strength because the wave is shadowed or blocked by the obstacle. The higher the frequency, the harder it becomes for the signal to penetrate an obstacle, which in this case is the vehicle since

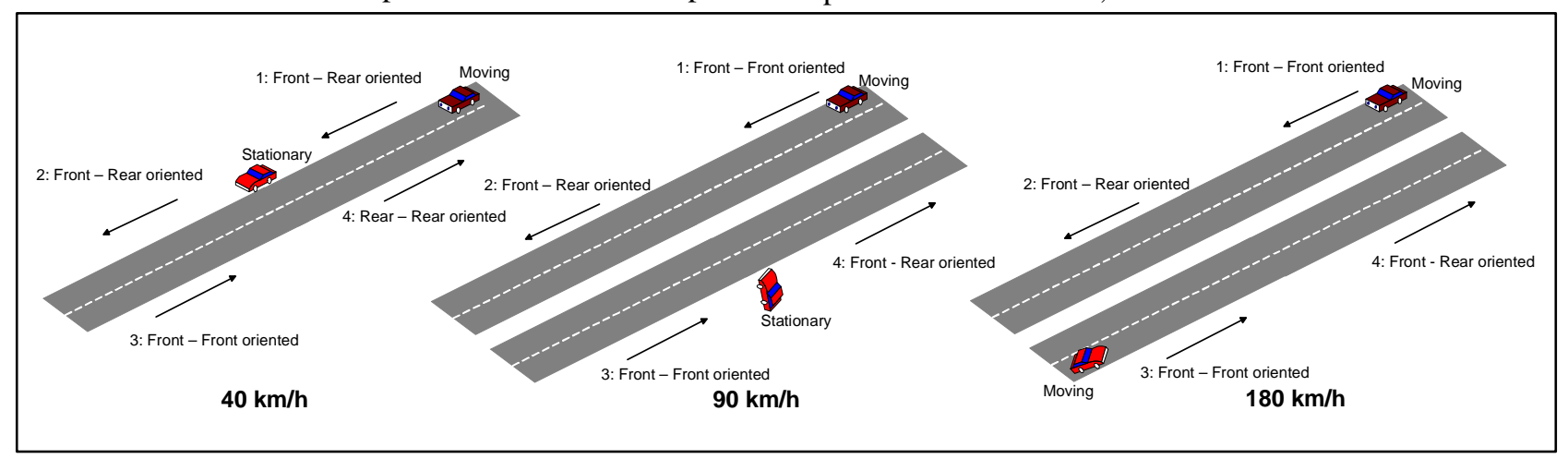

Figure 10: Performed link tests scenarios 
antennas are attached to the windscreen. This became very obvious during the experiments. In the left part of Figure 12 and Figure 13 it can be seen that the useful range is higher when vehicles approach each other (left part of the figure) oriented front to front than front to rear. Once the moving vehicle passed the parked vehicle in the middle of the figure, it can be seen that the PSR drops fast. From the figures it can be concluded that the .11g standard suffers from shadow fading in an acceptable way. However, the .11a standard can only be utilized between vehicles directly facing each other because it becomes practically unusable when signals have to penetrate the vehicle.

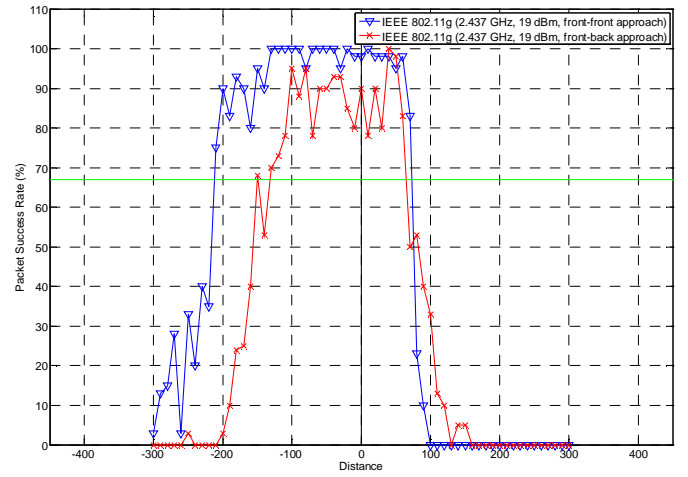

Figure 12: PSR different approaches $(40 \mathrm{~km} / \mathrm{u})$

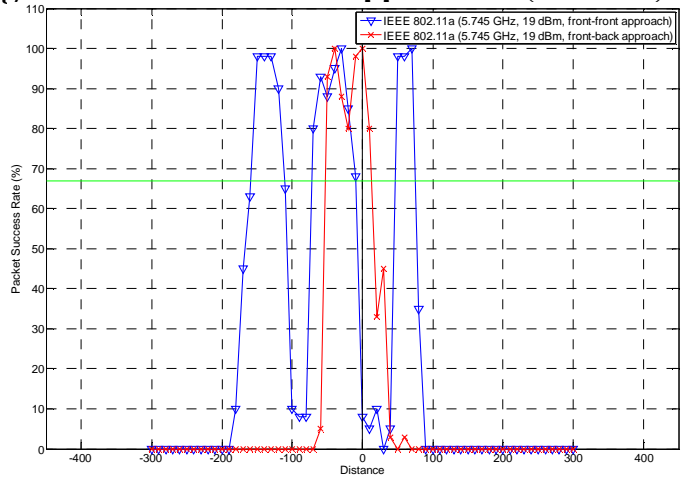

Figure 13: PSR different approaches $(40 \mathrm{~km} / \mathrm{u})$

\section{Multi-path fading}

Multi-path fading is a term used to describe signal distortion because of the multiple paths a radio wave may follow between transmitter and receiver due to reflections. At the receiver side this can cause significant inter-symbol interference (ISI) [12]. Both the $.11 \mathrm{~g}$ and .11a standard have a guard interval of $0.8 \mu \mathrm{s}$ to protect against ISI, but .11a is influenced more by multi-path fading than .11g because the higher the frequency of a signal becomes, the higher the probability becomes that it will reflect on a given surface. This is confirmed by Figure 11 where in exactly the same circumstances (low speed, front-front orientation, no other traffic) the PSR of $.11 \mathrm{~g}$ remains stable within its useful range, but .11a suffers from sudden drops. This was observed during multiple test runs, and can be explained as a consequence of multi-path fading.

\section{E. Doppler shift}

The Doppler shift is the change in frequency of a wave for an observer moving relative to the source of the wave [12]. It is commonly heard when a vehicle sounding a siren or horn approaches, passes, and recedes from an observer. In the case that the speed of the wave is much greater than the relative speed of the source and observer (e.g. with electromagnetic waves), the relationship between observed frequency and emitted frequency is symmetric. Doppler shift is proportional to the frequency: the higher the frequency becomes, the higher the Doppler shift becomes. This means that both $.11 \mathrm{~g}$ and $.11 \mathrm{a}$ suffer from reduced signal quality in case of high velocity, but the effect on the .11a standard is more significant. This is confirmed by Figure 14 and Figure 15. It can be seen that the .11g standard performs acceptably under high velocities, but .11a becomes practically unusable.

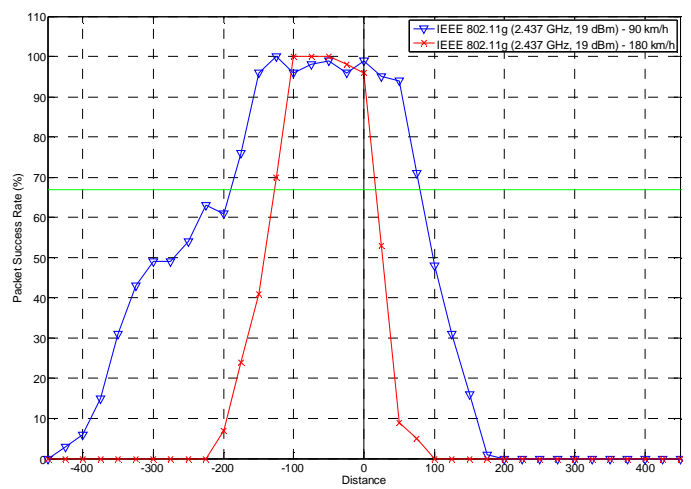

Figure 14: PSR higher speeds .11g

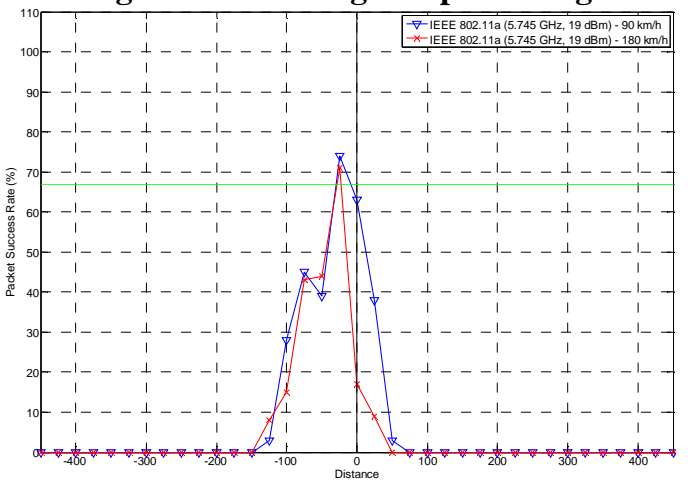

Figure 15: PSR higher speeds .11a

\section{F. Useful communication ranges of both standards}

The previous subsections provided valuable insights in the behavior of the $.11 \mathrm{~g}$ and $.11 \mathrm{a}$ standard in smartphone VANETs. To summarize, the useful communication range of $.11 \mathrm{~g}$ is larger than of .11a in line-of-sight conditions. Besides, the .11a standard has more difficulties with signals that have to penetrate a vehicle than the $.11 \mathrm{~g}$ standard, and in case of higher relative speeds the .11a standard becomes practically unusable while the $.11 \mathrm{~g}$ standard performs reasonably. Bearing these observations in mind, the feasibility of utilizing smartphones for VANETs can be determined based on the useful communication ranges of both the $.11 \mathrm{~g}$ and $.11 \mathrm{a}$ standard. We will focus on the 15 and $19 \mathrm{dBm}$ results because they are most representative of smartphones. The $23 \mathrm{dBm}$ can indicate if 
certain problems could be partially solved by utilizing standard interfaces with higher output powers.

As can be seen in Figure 16, at low speeds both standards provide acceptable ranges of $100-150 \mathrm{~m}$ when vehicles are oriented front to front. In the other orientations, $.11 \mathrm{~g}$ also performs acceptable $(50-100 \mathrm{~m})$ but the $.11 \mathrm{a}$ standards becomes unfeasible (under $50 \mathrm{~m}$ ). At a speed of $90 \mathrm{~km} / \mathrm{h}, .11 \mathrm{~g}$ maintains this behavior, but .11a becomes entirely unusable (under $50 \mathrm{~m}$ in all orientations). At a speed of $180 \mathrm{~km} / \mathrm{h} .11 \mathrm{~g}$ remains feasible, but only with a transmit power of $19 \mathrm{dBm}$, with a communication range of 150 meters in front-front orientation and 100 meters in rear-rear orientation.

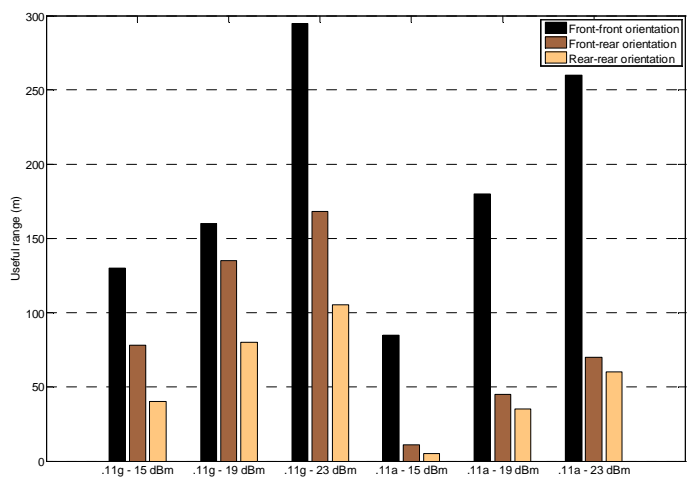

Figure 16: Useful communication range $(40 \mathrm{~km} / \mathrm{h})$

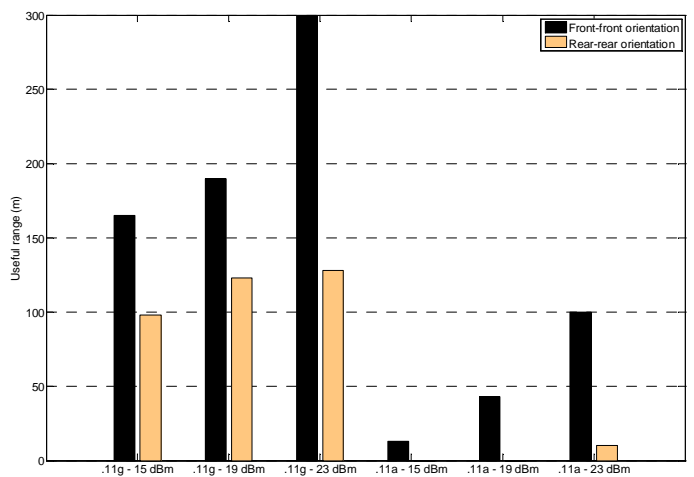

Figure 17: Useful communication range $(90 \mathrm{~km} / \mathrm{h})$

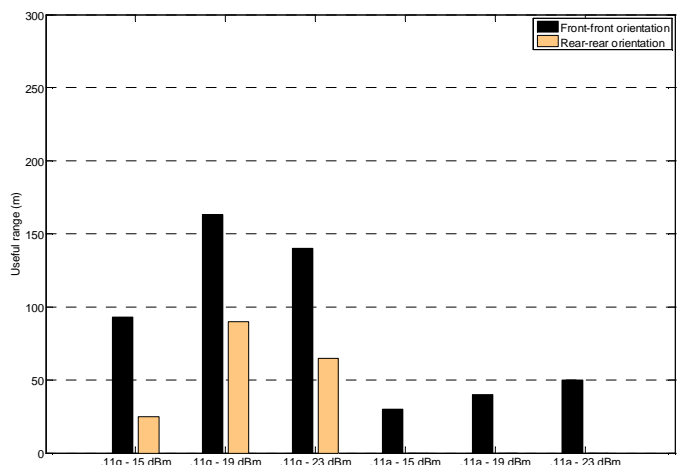

Figure 18: Useful communication range $(180 \mathrm{~km} / \mathrm{h})$

\section{CONCLUSION}

From a business point of view, utilizing smartphones for vehicular ad hoc networking is very attractive. From a technical point of view, this seems possible in specific circumstances. The presented results indicate that the utilization of smartphones for vehicular ad hoc networking is feasible, but only in highway environments using IEEE $802.11 \mathrm{~g}$ interfaces (which operate in the $2.4 \mathrm{GHz}$ band) with a maximum output power of at least $19 \mathrm{dBm}$. Although these preconditions seem quite restrictive, a significant amount of known cooperative road safety applications specifically targets the highway scenario. Therefore it can be concluded that the smartphone VANET approach could pave the way for the adoption of cooperative road safety applications by the general public.

\section{ACKNOWLEDGMENT}

The authors would like to thank David Carels for his assistance in the performance of the experiments described in section IV.

\section{REFERENCES}

[1] ETSI, "ETSI TR 102638 Intelligent Transport Systems (ITS); Vehicular Communications; Basic Set of Applications; Definitions", http://www.etsi.org, 2009

[2] G M. Bayly, B. Filde, M. Regan and K.Young, "View of crash effectiveness of Intelligent Transport Systems", Deliverable D4.1.1 D6.2, TRACE project, 2007

[3] H. Peters, k. Assing, "Market failures in relation to the deployment of cooperative vehicle safetey systems", Proc. ITS Europe 2008, Geneva, 2008

[4] RACC Automobile Club, "Stakeholder utility, data privacy and usability analysis and recommendations for operational guarantees and system safeguards: Europe", Deliverable D.DEPN.4.1, CVIS project, 2007

[5] The Nielsen Company, "Apple leads smartphone race, while android attracts most recent customers", internet article, http://blog.nielsen.com/nielsenwire/online mobile/apple-leadssmartphone-race-while-android-attracts-most-recent-customers/, last accessed May $24^{\text {th }} 2011$

[6] IEEE WG802.11 - Wireless LAN Working Group, “ 802.11 p-2010 IEEE Standard for Local and Metropolitan Area Networks - Specific requirements Part 11: Wireless LAN Medium Access Control (MAC) and Physical Layer (PHY) Specifications Amendment 6: Wireless Access in Vehicular Environments", http://standards.ieee.org, 2010

[7] ISO/TC 204, "ISO 21215:2010 Intelligent transport systems Communications access for land mobiles (CALM)-M5", http://www.iso.org, 2010

[8] L. Stibor, Y. Zang and H.-J. Reumerman, "Evaluation of communication distance of broadcast messages in a vehicular ad-hoc network using IEEE 802.11p", in Proc. Wireless Communications and Networking Conference (WCNC), Hong Kong, 2007, pp. 254-257.

[9] ETSI, "ETSI TS 102 637-2, Intelligent Transport Systems; Vehicular Communications; Basic Set of Applications; Part 2: Specification of Cooperative Awareness Basic Service",http://www.etsi.org, 2010.

[10] ETSI, "ETSI TS 102 637-3, Intelligent Transport Systems; Vehicular Communications; Basic Set of Applications; Part 2: Specification of Decentralized Environment Notification Basic Service", http://www.etsi.org, 2010.

[11] C.A. Balanis, "Antenna Theory", 2003, John Wiley and Sons Inc.

[12] J. Schiller, "Mobile communications 02 Edition", published by Pearson Eductation Limited, 2003. 